\title{
Anisoplanatism in adaptive optics systems due to pupil aberrations
}

B.J. Bauman

August 17, 2005

Optics and Photonics

San Diego, CA, United States

July 31, 2005 through August 4, 2005 
This document was prepared as an account of work sponsored by an agency of the United States Government. Neither the United States Government nor the University of California nor any of their employees, makes any warranty, express or implied, or assumes any legal liability or responsibility for the accuracy, completeness, or usefulness of any information, apparatus, product, or process disclosed, or represents that its use would not infringe privately owned rights. Reference herein to any specific commercial product, process, or service by trade name, trademark, manufacturer, or otherwise, does not necessarily constitute or imply its endorsement, recommendation, or favoring by the United States Government or the University of California. The views and opinions of authors expressed herein do not necessarily state or reflect those of the United States Government or the University of California, and shall not be used for advertising or product endorsement purposes. 


\title{
Anisoplanatism in adaptive optics systems due to pupil aberrations
}

\author{
Brian J. Bauman \\ Lawrence Livermore National Laboratory, 7000 East Avenue, Livermore, CA 94550 \\ Laboratory for Adaptive Optics, University of California, Santa Cruz, \\ 1156 High Street, Santa Cruz, CA 95064
}

\begin{abstract}
Adaptive optics systems typically include an optical relay that simultaneously images the science field to be corrected and also a set of pupil planes conjugate to the deformable mirror of the system. Often, in the optical spaces where DM's are placed, the pupils are aberrated, leading to a displacement and/or distortion of the pupil that varies according to field position - producing a type of anisoplanatism, i.e., a degradation of the AO correction with field angle. The pupil aberration phenomenon is described and expressed in terms of Seidel aberrations. An expression for anisoplanatism as a function of pupil distortion is derived, an example of an off-axis parabola is given, and a convenient method for controlling pupil-aberration-generated anisoplanatism is proposed.
\end{abstract}

Keywords: adaptive optics, deformable mirror, anisoplanatism, angular anisoplanatism, pupil aberration, pupil distortion, Kolmogorov, off-axis parabola

\section{OVERVIEW}

Section 2 describes the phenomenon of anisoplanatism due to pupil aberrations in an AO system. Section 3 describes the effect of pupil distortions in terms of anisoplanatism-like wavefront error penalties. Section 4 examines pupil distortions arising from various aberrations in the optical relay. Section 5 examines the specific case of an off-axis parabola (OAP) and shows that the anisoplanatism generated by pupil aberrations becomes rapidly more severe for small deformable mirrors (DM's) and large fields. Section 6 describes a method to minimize pupil aberrations in terms of easy-to-use and easy-to-optimize metrics.

\section{INTRODUCTION}

Single-conjugate astronomical adaptive optics (AO) systems typically include an optical relay between the telescope and the DM, and another relay between the DM and other pupils such as that in a wavefront sensor (WFS). Each relay performs dual functions. Primarily, the relays image the science field and guide stars (GS's) from the telescope focus to other image positions. Secondarily, the relays also form sets of planes conjugate to the DM of the system; the telescope pupil is often one of the planes conjugate to the DM.

As an example, following the cassegrain focus, the first element in the AO relay is commonly an OAP, which collimates the light from the science field and also produces a pupil at which the DM is located. In general the pupil will be aberrated since the OAP is oriented so that the parabola's focus is located at the center of the science field (in order to yield good imagery of the science field on-axis); see figure 1. A close view of the pupil reveals that the pupil is aberrated. In fact, in this case, each point in the pupil is aberrated into a comatic pattern (figure 2), which is not surprising given that the pupil is located off the optical axis.

Often, this pupil aberration is not a problem, but to the extent that the pupil aberrations exist, the system will suffer from a kind of anisoplanaticism. This is because the chief rays from the various field angles will not all pass through the center of the pupil (paraxially, the chief rays all pass through the center of the pupil, by definition, but for real rays, this is not generally true). In other words, the pupil (i.e., the DM and the correction it applies to the wavefront) is shifted with respect to the telescope pupil by an amount depending on the field angle - this is similar to anisoplanaticism caused by atmospheric aberrations at an altitude not conjugate to the DM (figure 3). The difference between the pupilaberration-derived anisoplanaticism and an altitude-derived anisoplanatism is in the form of the pupil error: with 
altitude-generated anisoplanaticism, the error is linear with field angle whereas that the pupil aberration-generated one is much more complex - it is a comatic pattern. The majority of the effect is to displace/shear the pupil, with the amount and direction of shear dependent on the field angle. There is also some pupil distortion, as evidenced by the fact that the three spot diagrams in figure 2 (left) are not exactly the same.

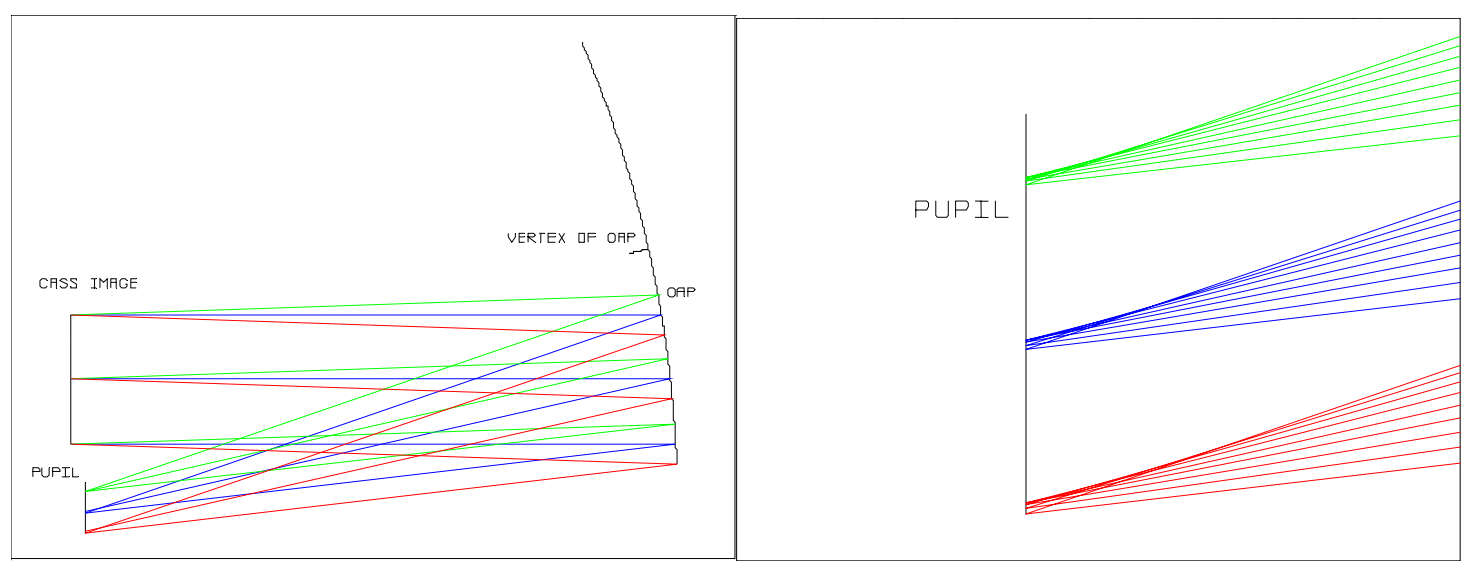

Figure 1: (left) Telescope cassegrain ("cass") image followed by an OAP which images a pupil. The telescope has a 30 meter diameter primary with a telecentric f/15 output. The optical axis of the parent parabola joins the indicated parabola vertex and the center of the cassegrain image. The pupil in the figure is $20 \mathrm{~mm}$ in diameter. Rays for on-axis (center of cass image) and \pm 15 arcsec off-axis (top and bottom of cass image) are shown with chief rays and top/bottom marginal rays. (right) zoomed-in view of region near pupil, showing a collection of chief rays for various field angles near the center of the figure, as well as corresponding collections of top and bottom marginal rays. The pupil is evidently aberrated, as detailed in figure 2. The non-normal incidence angle between the on-axis chief ray and the pupil plane is a manifestation of the OAP's Petzval curvature.

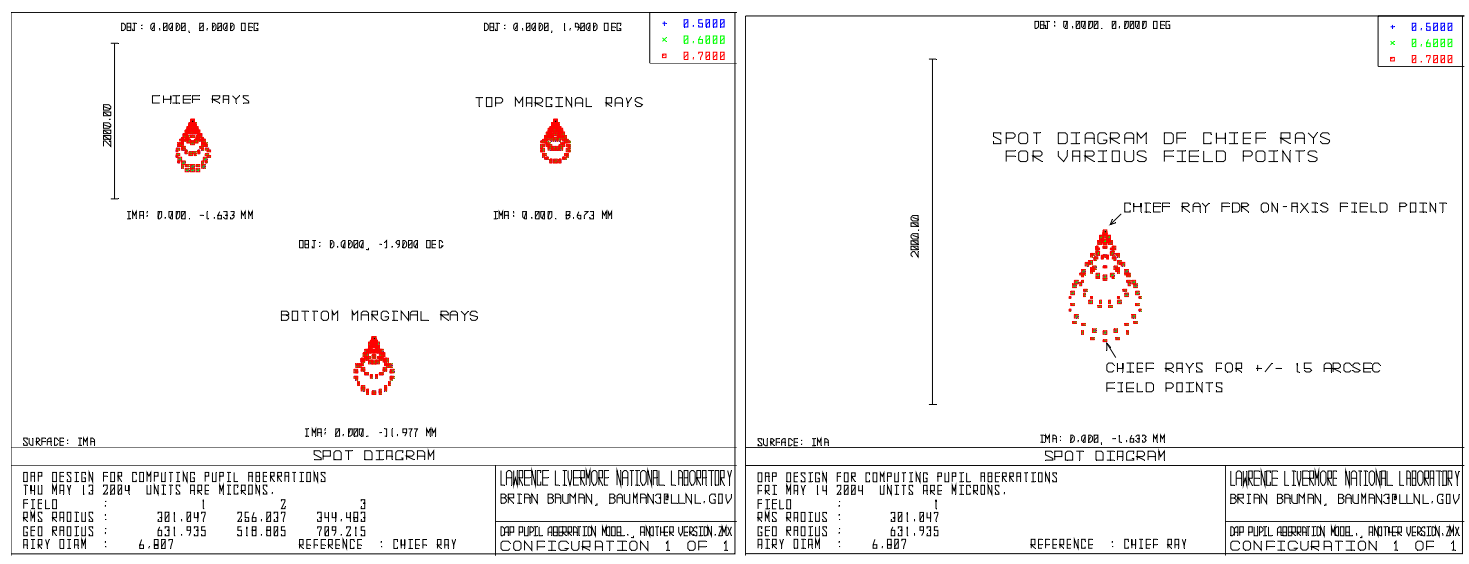

Figure 2: (left) Spot diagrams for 3 points in the pupil (center, top, and bottom of pupil). Each mark indicates a different field angle. (right) magnified view of spot diagram for chief rays only. The chief ray for the on-axis field point is at the top of the figure and the chief rays for \pm 15 aresec are at the bottom of the figure.

The pupil aberrations to be considered will produce anisoplanatism-like wavefront errors. In order to isolate this effect from other forms of anisoplanatism, it will be assumed that there is a single layer of Kolmogorov atmospheric aberration localized at the ground and that the DM is conjugate to that layer. Further, it will be assumed that all pupils are registered or calibrated for the on-axis field point so that the DM perfectly corrects the atmospheric aberrations on-axis. All other error terms, such as fitting error, temporal lag, and other signal-to-noise ratio concerns are assumed to be 
absent. This approach of analyzing one effect in isolation is consistent with that used to assess other terms in an AO performance error budget.

As indicated earlier, such a shift or distortion in the pupil for off-axis field points does in fact occur in AO optical relays. Taking a phenomenological approach, the pupil aberrations can be divided into three types: those that vary from position to position within the pupil, but do not vary with field angle on the sky (e.g., Seidel pupil distortion); those that vary according to field position, but are consistent from position to position within the pupil (e.g., pupil spherical aberration); and those that vary according to both pupil position and field position (e.g., pupil coma). The first type is a pure pupil distortion, such as a barrel distortion, which warps the first-order mapping between the DM and another pupil plane, such as the telescope aperture or the WFS. This does not necessarily lead to performance degradation. Since the pupil warping is consistent for all field points, the telescope/DM registration and DM/WFS registration work perfectly for all field points. There may be an effect on the point-spread function owing to the distortion of the pupil from a uniformly-illuminated circle (or whatever the shape of the aperture stop is), but that is not specifically an AO penalty.

It will be convenient to consider these pupil aberrations in terms of transverse ray aberrations. It will also be convenient at times to use the Seidel aberrations cast in terms of pupil imagery, such as pupil spherical aberration, pupil coma, and pupil distortion.

\section{ANISOPLANATIC WAVEFRONT ERROR DUE TO GENERAL PUPIL DISTORTIONS}

Before considering the lens design issues, we can consider the performance penalties due to a DM correction being applied in a shifted or distorted manner, as is the case for an off-axis field point. A transverse shift in the correction is familiar, as indicated earlier: it is equivalent to the anisoplanatic error due to an aberration localized to an altitude other than that for the DM. Qualitatively, one can expect significant image degradation if the pupil shift is comparable or greater than $r_{0}$, Fried's seeing parameter ${ }^{1}$ - where the pupil shift and $r_{0}$ are measured in the same optical space. Quantitatively, the expressions for angular anisoplanatism can be used to derive the anisoplanatism caused by pupil aberrations. The relationship is most clearly seen by considering the angular anisoplanatism equations in the case of a single thin layer of turbulence at a height $h$. The phase variance (in radians ${ }^{2}$ ) due to angular anisoplanatism is given by ${ }^{2}$

$$
\left\langle\underset{\substack{\text { anisoplanatism, } \\ \text { angular }}}{\sigma^{2}}(\theta)\right\rangle=\left(\frac{\theta}{\theta_{0}}\right)^{5 / 3}
$$

where $\theta$ is the field angle relative to the direction of perfect correction, and $\theta_{0}$ is the characteristic field angle at which the phase variance is 1 radian $^{2}$, known as the isoplanatic angle. The expectation value brackets will be dropped from this point forward. For a single layer, the well-known 5/3 power law can be viewed in terms of pupil shears, $s=h \theta$ and $s_{0}=h \theta_{0}$ as depicted in figure 3 .

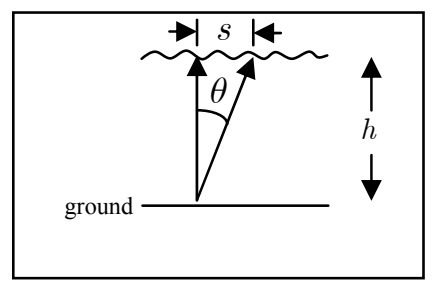

Figure 3: Sketch of the relationship between altitude, shear distance, and field angle for a layer of aberration at an altitude $h$.

Therefore, anisoplanatism due to pupil shear is equal to the $5 / 3$ power of the normalized pupil shear:

$$
\sigma_{\substack{\text { anisoplanatism, } \\ \text { pupilshear }}}^{2}(s)=\left(\frac{s}{s_{0}}\right)^{5 / 3}
$$


The amount of pupil shear corresponding to $1 \operatorname{radian}^{2}$ of phase variance can be shown ${ }^{3}$ to be $s_{0}=h \theta_{0}=0.314 r_{0}$. So,

$$
\sigma_{\substack{\text { anisoplanatism }, \\ \text { pupilshear }}}^{2}(s)=\left(\frac{s}{0.314 r_{0}}\right)^{5 / 3}
$$

An equation for wavefront variance due to an arbitrary pupil distortion will now be derived. Rather than return to "first principles" using structure functions for Kolmogorov turbulence, one can extend the anisoplanatism equations above. In a warped pupil, the pupil shear is different for each pupil point $(x, y)$ and for each field angle $\vec{\theta}$. In other words, $s(x, y ; \vec{\theta})$ describes how the pupil is remapped by the pupil aberrations for each field angle. The contribution to the phase variance of that pupil point's shear is proportional to the $5 / 3$ power of the shear, $[s(x, y ; \vec{\theta})]^{5 / 3}$. The total phase variance over the entire pupil is just sum of the phase variance contributions, integrated over the pupil, and normalized for the pupil area:

$$
\sigma_{\substack{\text { anisoplanatism, } \\ \text { pupil distortion }}}^{2}(\theta)=\frac{\int_{\text {pupil }}\left(\frac{s(x, y ; \vec{\theta})}{s_{0}}\right)^{5 / 3} d x d y}{\int_{\text {pupil }} d x d y}
$$

As a check, if the pupil is shifted by a distance $0.314 r_{0}$ that is constant across the pupil, then the equation yields 1 radian $^{2}$ of phase variance, as it should.

From this point forward, all references to anisoplanatism will refer to pupil aberration-derived anisoplanatism.

\section{PUPIL ABERRATIONS IN THE AO RELAY}

Equation 4 gives the AO system performance penalty from an arbitrary pupil distortion between the atmospheric aberration and correction. Now we can consider the pupil distortion between the ground layer and the DM that is generated by the AO relay. Suppose that the pupil aberrations can be described by the pupil Seidel aberrations. Employing the "W" notation used by Welford ${ }^{4}$ and Shack's vector extension of the aberration expressions ${ }^{5}$ :

\begin{tabular}{|l|l|l|}
\hline Aberration & Wavefront form & Ray form \\
\hline $\begin{array}{l}\text { Pupil } \\
\text { spherical } \\
\text { aberration }\end{array}$ & $\bar{W}_{040}(\vec{H} \cdot \vec{H})^{2}$ & $\frac{4 \bar{W}_{040}}{\omega^{\prime}}(\vec{H} \cdot \vec{H}) \vec{H}=\underbrace{\frac{\bar{W}_{040}}{\omega^{\prime}} H^{3} \widehat{H}}_{\begin{array}{c}\text { pupil shiff } \propto H^{3} \\
\text { in direction of field }\end{array}}$ \\
$=\bar{W}_{040} H^{4}$ & \\
\hline
\end{tabular}




\begin{tabular}{|c|c|c|}
\hline Pupil coma & $\begin{array}{l}\bar{W}_{131}(\vec{\rho} \cdot \vec{H})(\vec{H} \cdot \vec{H}) \\
=\bar{W}_{131} \rho H^{3} \cos \phi\end{array}$ & $\begin{aligned} & \frac{\bar{W}_{131}}{\omega^{\prime}}[(\vec{H} \cdot \vec{H}) \vec{\rho}+2(\vec{\rho} \cdot \vec{H}) \vec{H}] \\
= & \frac{\bar{W}_{131}}{\omega^{\prime}}(\underbrace{\rho H^{2} \hat{\rho}}_{\begin{array}{l}\text { radial pupil } \\
\text { expansion, } \propto H^{2}\end{array}}+\underbrace{2 \rho H^{2} \cos \phi \widehat{H}}_{\begin{array}{l}\text { pupil stretching } \\
\text { in direction of field } \\
\propto H^{2}\end{array}}\end{aligned}$ \\
\hline $\begin{array}{l}\text { Pupil } \\
\text { astigmatism }\end{array}$ & $\begin{array}{l}\bar{W}_{222}(\vec{\rho} \cdot \vec{\rho})(\vec{H} \cdot \vec{H}) \\
=\bar{W}_{222} \rho^{2} H^{2} \cos ^{2} \phi\end{array}$ & $\frac{2 \bar{W}_{222}}{\omega^{\prime}}(\vec{H} \cdot \vec{\rho}) \vec{\rho}=\underbrace{\frac{2 \bar{W}_{222}}{\omega^{\prime}} \rho^{2} H \cos \phi \hat{\rho}}_{\substack{\text { quadratic radial stretching, } \\
\text { maximum parallel to field, } \\
\propto H}}$ \\
\hline $\begin{array}{l}\text { Pupil field } \\
\text { curvature }\end{array}$ & $\begin{array}{l}\bar{W}_{220}(\vec{\rho} \cdot \vec{\rho})(\vec{\rho} \cdot \vec{H}) \\
=\bar{W}_{220} \rho^{3} H \cos \phi\end{array}$ & $\frac{\bar{W}_{220}}{\omega^{\prime}}(\vec{\rho} \cdot \vec{\rho}) \vec{H}=\underbrace{\frac{\bar{W}_{220}}{\omega^{\prime}} \rho^{2} H \widehat{H}}_{\begin{array}{c}\text { quadratic pupil stretching } \\
\text { in direction of field, } \propto H\end{array}}$ \\
\hline $\begin{array}{l}\text { Pupil } \\
\text { distortion }\end{array}$ & $\begin{array}{l}\bar{W}_{311}(\vec{\rho} \cdot \vec{\rho})(\vec{\rho} \cdot \vec{\rho}) \\
=\bar{W}_{311} \rho^{4}\end{array}$ & $\frac{\bar{W}_{311}}{\omega^{\prime}}(\vec{\rho} \cdot \vec{\rho}) \vec{\rho}=\underbrace{\frac{\bar{W}_{311}}{\omega^{\prime}} \rho^{3} \hat{\rho}}_{\begin{array}{c}\text { cubic radial } \\
\text { pupil distortion, } \\
\text { independent of field }\end{array}}$ \\
\hline
\end{tabular}

Table 1: Pupil aberrations expressed in wavefront and ray aberration forms, with interpretations.

$\vec{H}$ indicates normalized field position (on the sky), $\vec{\rho}$ indicates normalized pupil position, and $\widehat{H}$ and $\widehat{\rho}$ indicate the corresponding unit vectors. $\vec{H}$ and $\vec{\rho}$ are defined within the unit circle, and $H$ and $\rho$ indicate the magnitudes of the corresponding vectors. $\phi$ is the angle between $\vec{H}$ and $\vec{\rho}$. $\omega^{\prime}$ is the marginal ray slope. The subscripts on the $W$ 's denote the various types of wavefront aberration, as described by Welford: $W_{\alpha \beta \gamma}$ is the coefficient of the term $H^{\alpha} \rho^{\beta} \cos ^{\gamma} \phi$; for example, $W_{131}$ indicates coma. The overbars on the $W$ 's denote pupil aberration coefficients rather than image aberration coefficients.

Pupil ray aberrations that are proportional to $\vec{\rho}=\rho \hat{\rho}$ amount to an undistorted pupil expansion. If the pupil ray aberration is proportional to a higher power of $\rho$ or is dependent on $\phi$, then the pupil becomes distorted. Pupil ray aberrations with a factor of $\widehat{H}$ indicate shifts and distortions parallel to the direction of the field. Except for pupil distortion, the pupil aberrations evaluate to zero for an on-axis field position. The pupil distortion does not matter since it is constant with field position, and so if we take pupil aberrations to be with respect to that obtained at the on-axis field position, then we can ignore the pupil distortion term. This is consistent with our notion that the pupil registrations are performed on-axis.

The various pupil aberrations in table 1 generate fairly straightforward pupil aberrations. Although the fractional power of the shear in equation 4 makes closed-form analysis difficult, it does not impede numerical analysis. Just as importantly, table 1 allows one to gain a sense for how the pupil aberrations are distorting the pupil for various field positions. Section 6 will propose a method for simplifying the problem for purposes of optimization in optical design.

When a pupil is located off the optical axis of the relay, as is usually the case with an off-axis parabola, then additional pupil aberration terms are generated ${ }^{6}$. Briefly, each instance of $\vec{\rho}$ above is replaced by $\overrightarrow{\rho_{0}}+\vec{\rho}$, where $\overrightarrow{\rho_{0}}$ indicates the 
normalized off-axis vector. As mentioned earlier, pupil distortion does not depend on field angle, so we can omit it here. Additional angles need to be defined: $\phi_{0}$ is the angle between $\vec{\rho}_{0}$ and $\vec{H} ; \psi$ is the angle between $\vec{\rho}$ and $\overrightarrow{\rho_{0}}$.

\begin{tabular}{|c|c|c|c|}
\hline Aberration & \multicolumn{3}{|l|}{ Ray form } \\
\hline $\begin{array}{l}\text { Pupil } \\
\text { spherical } \\
\text { aberration }\end{array}$ & \multicolumn{3}{|l|}{$\frac{4 \bar{W}_{040}}{\omega^{\prime}}(\vec{H} \cdot \vec{H}) \vec{H}=\underbrace{4 \frac{\bar{W}_{040}}{\omega^{\prime}} H^{3} \widehat{H}}_{\begin{array}{c}\text { pupil shift in direction } \\
\text { of fiedd, } \sim H^{3} \text { same as } \\
\text { on-axis case }\end{array}}$} \\
\hline Pupil coma & \multicolumn{3}{|c|}{$\begin{array}{l}\frac{\bar{W}_{131}}{\omega^{\prime}}\left[(\vec{H} \cdot \vec{H})\left(\overrightarrow{\rho_{0}}+\vec{\rho}\right)+2\left(\left(\overrightarrow{\rho_{0}}+\vec{\rho}\right) \cdot \vec{H}\right) \vec{H}\right] \\
=\frac{\bar{W}_{131}}{\omega^{\prime}} \underbrace{\rho_{0} H^{2} \widehat{\rho_{0}}}_{\begin{array}{l}\text { pupil shift in } \\
\text { off-axis direction, } \\
\propto H^{2}\end{array}}+\underbrace{\rho_{0} H^{2} \cos \phi_{0} \widehat{H}}_{\begin{array}{l}\text { pupil shift } \\
\text { in direction of field, } \\
\text { maximum in off-axis } \\
\text { direction, } \propto H^{2}\end{array}})+0\end{array}$} \\
\hline $\begin{array}{l}\text { Pupil } \\
\text { astigmatism }\end{array}$ & \multicolumn{3}{|c|}{ 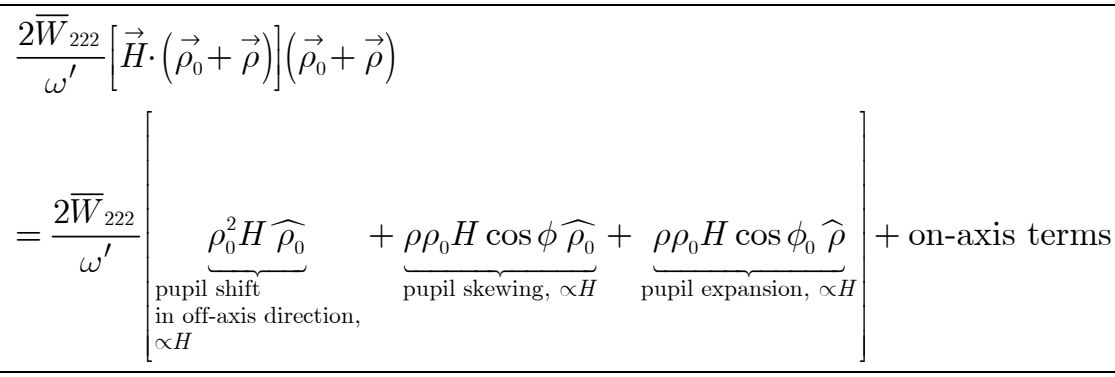 } \\
\hline $\begin{array}{l}\text { Pupil field } \\
\text { curvature }\end{array}$ & $=\frac{\bar{W}_{220}}{\omega^{\prime}} \mid \underbrace{\rho_{0}^{2} H \widehat{H}}_{\begin{array}{l}\text { pupil shift } \\
\text { in direction } \\
\text { of field, } \propto H\end{array}}+\underbrace{2 \rho \rho_{0} \cos \psi H \widehat{H}}_{\begin{array}{l}\text { pupil skewing } \\
\text { in direction } \\
\text { of field, } \propto H\end{array}}$ & + on-axis terms & \\
\hline
\end{tabular}

Table 2: Ray form of off-axis pupil aberrations, with interpretations.

\section{EXAMPLE OF AN OFF-AXIS PARABOLA}

The most common AO relay between the telescope and DM plane is an OAP, as shown in figure 1. This section gives results for the case of a 30 meter diameter, $\mathrm{f} / 15$ telescope with various field sizes and pupil/DM sizes. The OAP collimates the light and creates a pupil, and by changing the focal length, one can obtain various pupil/DM sizes. The off-axis distance of the OAP is determined for this case study by setting a minimal separation of $25 \mathrm{~mm}$ between the cassegrain image and the edge of the pupil/DM.

Figure 3 shows the maximum pupil shift with the field diameter in terms of percentage of $\mathrm{r}_{0}$, assuming $\mathrm{r}_{0}=50 \mathrm{~cm}$. In other words, the plot gives the pupil shift for the worst-performing field position within the designated field diameter. Figure 4 displays the same data in terms of anisoplanatic rms wavefront error, using the equation 4. 
It is immediately obvious that the pupil aberrations could become problematic for large fields and small DM's. A configuration with a 20 arcsec diameter field and $30 \mathrm{~mm}$ diameter pupil would result in as much as $\sim 200 \mathrm{~nm} \mathrm{rms}$ wavefront error, which can consume the entire error budget of an AO system. For a 30 arcsec field, the DM would need to be at least $60 \mathrm{~mm}$ in diameter for a more tolerable $60 \mathrm{~nm}$ rms error. Note that for this level of anisoplanatism and a telescope plate scale of $2.2 \mathrm{~mm} / \operatorname{arcsec}$ (30 meter, $\mathrm{f} / 15$ telescope), the linear field size and the DM size are comparable.
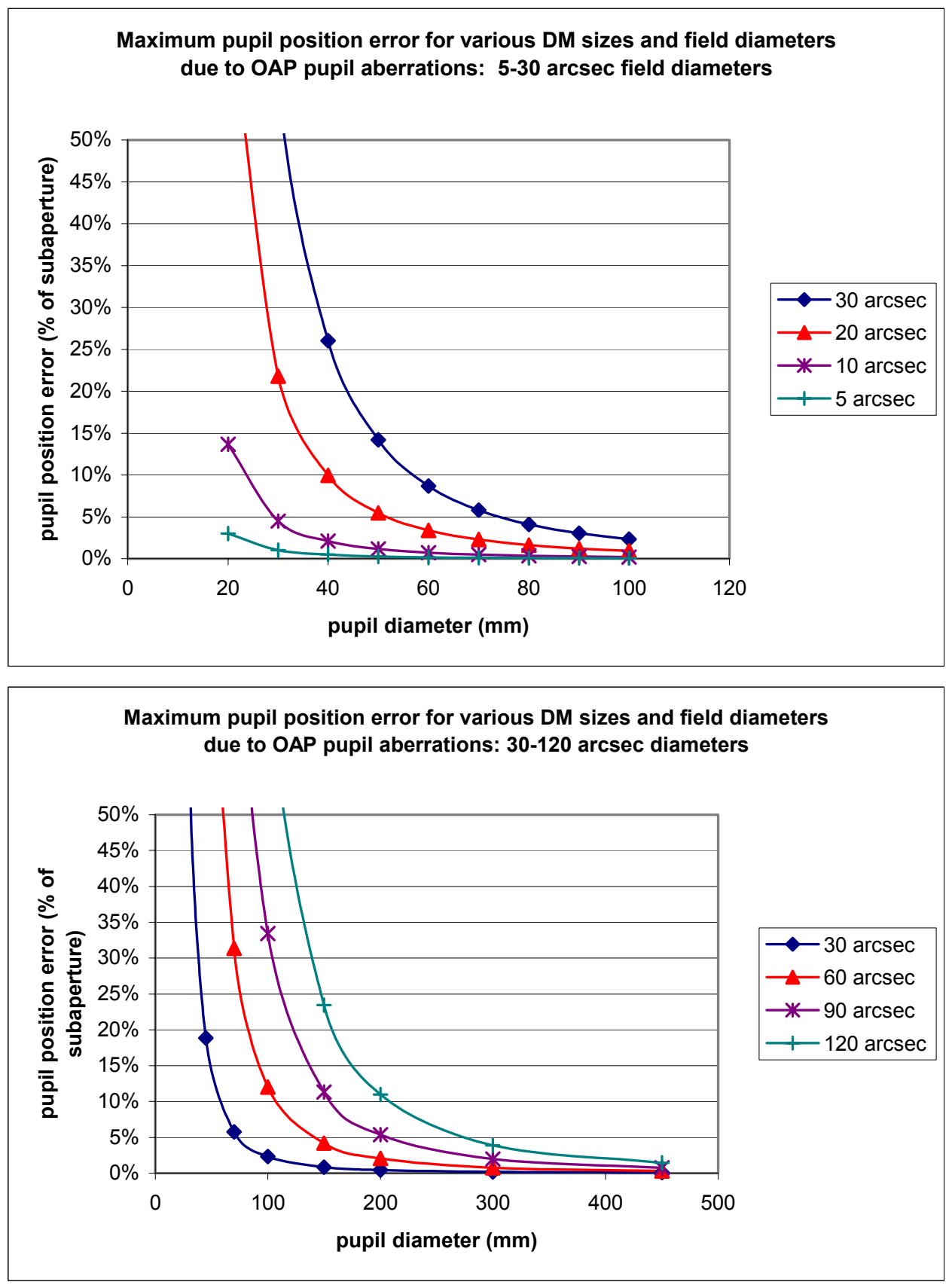

Figure 4: (top) Maximum error of pupil position for various DM sizes and field diameters (5-30 arcsec). For a given system (i.e., a given telescope $\mathrm{f \# ,} \mathrm{field} \mathrm{diameter,} \mathrm{and} \mathrm{pupil} \mathrm{diameter),} \mathrm{the} \mathrm{chief} \mathrm{rays} \mathrm{for} \mathrm{the} \mathrm{various} \mathrm{field} \mathrm{points} \mathrm{are} \mathrm{aberrated} \mathrm{by}$ various amounts and directions. This plot shows the maximum such aberration for a given system. $\mathbf{r}_{0}$ is assumed to be $50 \mathrm{~cm}$. (bottom) Same as top, but for larger DM sizes and field diameters (30-120 arcsec). Note change of horizontal scale. 

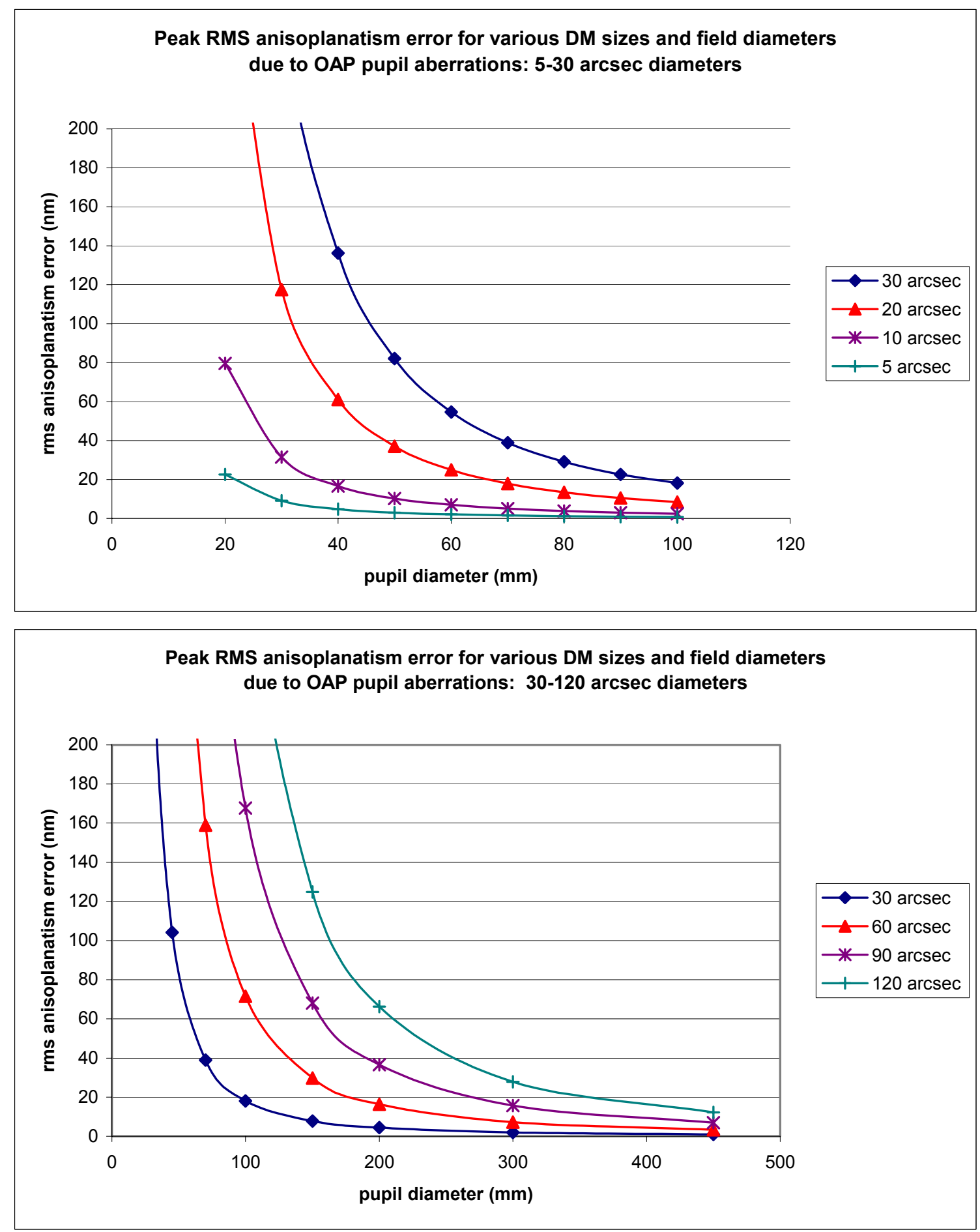

Figure 5: (top) Peak error of pupil position for various DM sizes and field diameters (from 5 to 10 arcsec), expressed in terms of anisoplanatic rms wavefront error. "Peak" refers to the field point that has the greatest pupil shear. $r_{0}$ is assumed to be $50 \mathrm{~cm}$. (bottom) Same as top, but for larger DM sizes and field diameters (30-120 arcsec). Note change in horizontal scale. 
One may wonder why this problem is not seen in current systems. Certainly AO systems with large, conventional DM's are less prone to this problem, but the smaller number of $r_{0}$ 's across the diameter of the DM helps greatly. The next generation of $\mathrm{AO}$ instruments will have large numbers of subapertures, which forces a much tighter tolerance for pupil aberrations in terms of percentage of the pupil diameter. For example, a shift of $1 \%$ of the pupil diameter is not problematic for a system with $\sim 10$ subapertures across the diameter (current systems), but is ruinous for a system with $\sim 100$ subapertures across the diameter (future systems).

\section{MINIMIZING PUPIL ABERRATIONS IN THE OPTICAL DESIGN}

Section 4 describes what pupil aberrations are generated by an AO relay. Section 3 describes how to apply those pupil distortions in order to generate an anisoplanatism penalty. Section 5 gives a specific example applying sections 3 and 4. The purpose of this section is to propose guidance in how to minimize these penalties in a practical optical design.

In computer-optimizing an optical design for science field imagery and for "pupil imagery" (to reduce anisoplanatism), one can set up two configurations in the lens file - one for each imaging mode. A metric that can be used for evaluating pupil imagery is the image spot size of various pupil points. Reducing this metric, of course, reduces the overall anisoplanatism of the system, but the metric does not give a measure of anisoplanatism at a given field angle, which can be helpful in trading performance in one portion of the field versus another portion, i.e., field-weighted optimization. To measure the anisoplanatism for a given field angle, one would measure the image displacement of many pupil points (with respect to their corresponding positions for an on-axis field point), and then insert those displacements into equation 4.

To gain some intuition, consider an optical space with an optical surface at left, with image and pupil planes at right; see figure 6, top left. The optical system here is unaberrated, i.e., accurately described by its first-order properties. Therefore the top marginal rays for all image points intersect at the top edge of the pupil; likewise for the bottom marginal rays. Also, the marginal rays for each field point would intersect at the paraxial image location for that field point.

Now suppose that we introduce an aberration at the optical surface (figure 6, top right). The ray height at the optical surface has not changed, but the ray has deviated so that there is a ray error at the image surface of $\varepsilon_{\text {image. }}$ By geometry, we can see that there is also a ray error at the pupil plane, $\varepsilon_{\text {pupil }}$ which is related to the $\varepsilon_{\text {image: }}$

$$
\frac{\varepsilon_{\text {pupil }}}{z_{\text {pupil }}}=\frac{\varepsilon_{\text {image }}}{z_{\text {image }}}
$$

When either the image distance or pupil distance becomes infinite or zero, the ray error may instead be expressed as an angle, $u=\varepsilon / z$.

As an important example, if the optical system can be adequately described in terms of its intrinsic third-order aberrations, then the ray heights are those predicted by first-order optics. The pupil ray errors are then proportional to the image ray errors in the same optical space. This is a powerful statement that could aid us in the optical design of the relay.

Now, if a previous optical surface has caused the marginal ray to strike the optical surface at a height other than that indicated by first-order optics, but the image is otherwise unaberrated, then a pupil ray error is generated as well, as shown at the bottom of figure 6 . The relationship is

$$
\frac{\varepsilon_{\text {pupil }}}{\varepsilon_{\text {lens }}}=\frac{z_{\text {image }}-z_{\text {pupil }}}{z_{\text {image }}}
$$




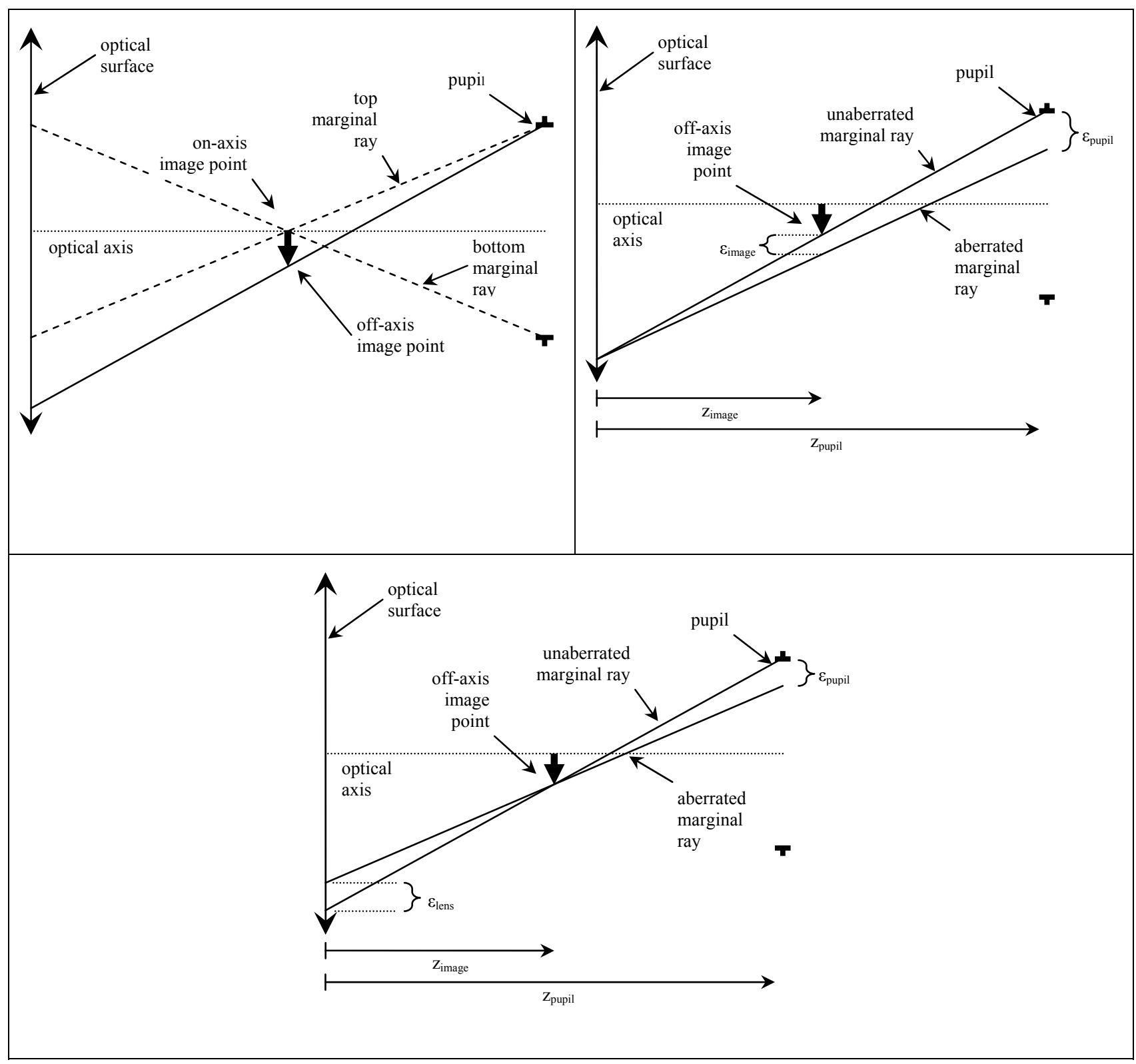

Figure 6: (top left) Layout of an optical space with an optical surface, and aberration-free image and pupil. The downward facing arrow represents the science object with on-axis and off-axis field points.

(top right) Same optical space as top, except that the top marginal ray for an off-axis field point is aberrated. The ray leaves the optical surface at the same position as in figure 3, but is aberrated at the image plane, and therefore aberrated at the pupil plane. The $z$ 's represent the distances from the optical surface to the image and pupil planes, and are positive when those planes are to the right of the optical surface. As shown, the $\varepsilon$ 's are negative.

(bottom) Same optical space as top, except that the top marginal ray leaves the optical surface at a different position, but is unaberrated at the off-axis field point. The ray is aberrated at the pupil plane. As shown, the $\varepsilon_{\text {lens }}$ is positive and $\varepsilon_{\text {pupil }}$ is negative. 
Intuitively, it can be seen that these errors may become significant if the induced ray height error is a significant fraction of the beam size at the optical surface. For example, if there were 100 subapertures across the beam and the induced ray height error at the optic were $1 \%$, then the pupil ray error would be $1 \%$ of the beam $=1$ subaperture, which would be significant. In a similar sense, ray angle errors in equation 5 would be equally significant if they are $1 \%$ of the numerical aperture.

The total pupil ray error for a given ray can be estimated by summing the two sources of pupil ray errors in equations 5 and 6. Since the ray errors are actually vectors (although not denoted as such as above), adding the errors means adding them in a vector sense.

If we can neglect the induced aberrations, then the pupil ray errors are proportional to the image ray errors, as indicated in equation 5. Looking at equation 4 , the phase variance due to pupil ray errors is the integral of the $5 / 3$ power of the ray errors. If we substitute a power of 2 in place of the power of $5 / 3$ (a rough approximation, of course, but useful, as shall be seen), then the phase variance is proportional to the variance of the pupil ray errors.

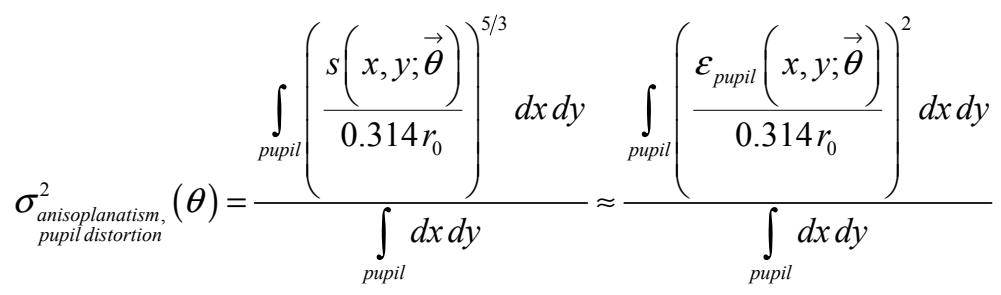

Since pupil ray errors are proportional to image ray errors (by equation 5), the anisoplanatic phase variance at a given field position due to pupil aberrations is proportional to the variance of the ray aberrations for that field position. Expressed in root-mean-square terms, the anisoplanatic rms phase error due to pupil aberrations is proportional the rms spot size for the field point and optical space in question (equation 8). This is a perhaps the most common metric used in lens design optimization!

$$
\begin{aligned}
\sigma_{\text {anisoplanatism, }}(\theta) & \approx \frac{1}{\text { pupildistortion }}\left(\frac{z_{\text {pupil }}}{z_{\text {image }}}\right)\left\{\frac{\int_{\text {pupil }}\left(\varepsilon_{\text {image }}(x, y ; \vec{\theta})\right)^{2} d x d y}{\int_{\text {pupil }} d x d y}\right\}^{\frac{1}{2}} \\
& \approx\left(\frac{\varepsilon_{\text {rms }, \text { image }}}{0.314 r_{0}}\right)\left(\frac{z_{\text {pupil }}}{z_{\text {image }}}\right)
\end{aligned}
$$

An implication is that one should place deformable mirrors in optical spaces which have well-corrected images. This is not commonly done, however. Often, the DM will be in collimated space, i.e., where the image is located at infinity, and in those locations, the aberrations are typically not balanced - they are balanced after the next parabola leading to an image plane. There are no requirements that a DM be placed in collimated space, but it is often convenient to do so. However, as DM sizes becomes small and subaperture counts become large, it may become necessary to change this design paradigm.

This is consistent with the problems illustrated in the OAP example in section 5. In the pupil-forming space following the OAP, the science field is significantly aberrated (primarily with coma) off-axis, and so it is not surprising to see that the pupil is similarly aberrated. The corrected field can be increased by adding a second element to the relay. If one desires collimated space for the pupil, then one can use, for example, an off-axis portion of a Gregorian-style (i.e., with intermediate image) Ritchey-Chrétien telescope. 
In fact, previous work ${ }^{7,8,9,10}$ suggest that optical spaces with well-corrected images also have well-corrected pupils. Future articles will integrate existing pupil aberration theory with the present application.

\section{CONCLUSIONS}

Anisoplanatism arising from pupil aberrations may become a significant issue in the design of future AO systems, particularly those utilizing small DM's and large fields. Expressions describing and interpreting those pupil aberrations, as well as the anisoplanatism penalties for general pupil distortions have been derived. It may be possible to express and control the anisoplanatism in terms of image aberrations, and it is proposed that this anisoplanatism may be reduced by placing DM's in optical spaces with well-corrected images.

\section{ACKNOWLEDGEMENTS}

This work was performed under the auspices of the U.S. Department of Energy by University of California, Lawrence Livermore National Laboratory under Contract W-7405-Eng-48.

This work was supported in part by the National Science Foundation Science and Technology Center for Adaptive Optics (CfAO), managed by the University of California at Santa Cruz under cooperative agreement No. AST-9876783, and in part by the Gordon and Betty Moore Foundation through its grant to the UCO/Lick Laboratory for Adaptive Optics.

\section{REFERENCES}

1. D.L. Fried " Optical resolution through a randomly inhomogeneous medium for very long and very short exposures", JOSA, 56, 1372-1379, 1966.

2. D.L. Fried, "Anisoplanatism in adaptive optics", JOSA A, 69, 399-406, 1982.

3. J.W. Hardy, Adaptive Optics for Astronomical Telescopes, 102-103, Oxford University Press, New York, 1998.

4. W.T. Welford, Aberrations of Optical Systems, Adam Hilger, Bristol, 1989.

5. R.V. Shack, Lectures in Optical System Design, Optical Sciences Center, University of Arizona, 1990.

6. K.P. Thompson, "Description of the third-order optical aberrations of near-circular pupil optical systems without symmetry", JOSA A, 22, 1389-1401, 2005.

7. C.G. Wynne, "Primary Aberrations and Conjugate change", Proceedings of the Physical Society of London, Section B, 65, 429-437, 1952.

8. D. Shafer, "Aberration theory and the meaning of life", International Lens Design Conference (1985), D.T. Moore, W.H. Taylor, eds., 554, SPIE, 25-30, Cherry Hill, NJ, 1985.

9. J.M. Hoffman, Induced aberrations in optical systems, Ph.D. dissertation, University of Arizona, 1993.

10. M.T. Chang and R.R. Shannon, "Pupil aberrations in zoom systems", Zoom Lenses II, E.I. Betensky, A. Mann, I.A. Neil, eds., 3129, SPIE, 205-216, San Diego, 1997. 